\title{
CONCIENCIA AMBIENTAL DE LOS HABITANTES DE LA COLONIA EMILIO PORTES GIL EN LA H. MATAMOROS, TAMAULIPAS
}

\author{
CATALINA VARGAS RAMOS ${ }^{1}$, CYNTHIA DINORAH BRIONES RAMÍREZ ${ }^{2}$, \\ MARÍA DEL PERPETUO SOCORRO MANCHA SÁNCHEZ ${ }^{3}$, PATRICIA \\ GUADALUPE MÚZQUIZ NOVOA ${ }^{4}$, ANTONIO VARGAS GAXIOLA ${ }^{5}$
}

Recibido el 10 de junio de 2013 y aprobado el 24 de junio de 2013

\section{RESUMEN}

La Conciencia ambiental en México es un tema de gran relevancia ya que las alteraciones de hoy en día sobre el medio ambiente son principalmente por actividades antropogénicas ya sea por el desarrollo industrial, consumismo, la contaminación, residuos sólidos, etc., por lo que es de gran importancia lograr un equilibrio entre la naturaleza - hombre y para alcanzar este equilibrio es necesario la educación ambiental la cual permite emplear diferentes estrategias para lograr generar sentimientos, desarrollar conciencia ambiental y evidenciar la necesidad de elaborar nuevos valores a partir de las experiencias directas. Por lo que la presente investigación tuvo como objetivo evaluar la Conciencia Ambiental de los habitantes de la colonia Emilio Portes Gil en la H. Matamoros (Tamaulipas) mediante el instrumento llamado Cuestionario de Actitudes Ambientales (CAAM) de Núevalos a una población de 50 habitantes conformada con $52 \%$ de mujeres y el $48 \%$ de hombres en un rango de edad entre los 20 a 31 años, obteniéndose una conciencia ambiental muy parecida con un $32 \%$ y $30 \%$ tanto del sexo femenino como del masculino respectivamente.

\section{PALABRAS CLAVE}

Conciencia ambiental, actitud ambiental, educación ambiental, valores ambientales.

\section{ENVIRONMENTAL AWARENESS OF THE THE EMILIO PORTES GIL COLONIA INHABITANTS IN H. MATAMOROS, TAMAULIPAS}

\begin{abstract}
Environmental awareness in Mexico is a very important issue since today's alterations on the environment are basically produced by anthropogenic activities either because of industrial development, consumerism, pollution, solid waste, etc.., reason why it is very important to achieve a balance between nature and man and, in order to achieve this balance, environmental education which can employ different strategies for generating feelings, developing environmental awareness and highlighting the need to develop new values from direct experiences is necessary. For this reason, this investigation has as its main objective the assessment of Emilio Portes Gil Colonia in H. Matamoros (Tamaulipas) inhabitants' Environmental Awareness, using Nuévalos' instrument called Environmental Attitudes Questionnaire (CAAM) to a population of 50 inhabitants conformed by $52 \%$ women and $48 \%$ men in an age range between 20 to 31 years, yielding a very similar environmental awareness with $32 \%$ and $30 \%$ of both the male and the female respectively.
\end{abstract}




\section{KEY WORDS}

Environmental awareness, environmental attitude, environmental education, environmental values.

\section{INTRODUCCIÓN}

La Conciencia Ambiental en México, se enlaza principalmente a los antiguos pobladores de estas tierras, quienes creían que el cuidado de la naturaleza era muy importante, incluso hasta los llamaban dioses, como el dios del agua, el dios de la tierra, entre otros, pero en las últimas décadas las alteraciones sobre el medio ambiente son principalmente por actividades antropogénicas como la tecnología o el desarrollo industrial. Este desarrollo industrial tiende a producir cada vez más por el consumismo de la sociedad y la falta de respeto hacia el medio ambiente, por lo que nos trae como consecuencia mayor consumo de recursos naturales, mayor contaminación, mayor residuo sólido, llevándonos a una problemática ambiental afectando la calidad de vida de los habitantes, por lo que es necesario lograr un equilibrio naturaleza-hombre (Vargas et al., 2010).

Para poder alcanzar este equilibrio, surge la educación ambiental, que según Espejel y Castillo (2008) es un elemento fundamental para crear conciencia, valores y hábitos para el cuidado del medio ambiente.

La educación ambiental da inicio con el principio 19, en la Conferencia de las Naciones Unidas sobre el Medio Humano en 1972 (Moreno, 2008), donde se plantea la importancia de la educación ambiental, por lo cual permite emplear diferentes estrategias relacionadas con experiencias directas (Molano, 2003).

La principal misión de la educación ambiental es la formación de un vínculo entre los valores, conocimientos, habilidades, para permitir a los individuos la unión al grupo al que pertenece.

Entonces, la solución es encontrar y emplear estrategias para llevar a cabo conocimientos y valores, por consiguiente desarrollar habilidades, por lo que la educación ambiental se plantea como una solución principal para la unión entre naturaleza-hombre (Acebal, 2010), ya que este es el principal responsable de estos grandes cambios que han surgido en el medio ambiente como se mencionó anteriormente (Ávila, s.f.).

Por consiguiente, el planeta necesita que la sociedad actué para poder encontrar soluciones ante estos problemas, y para poder alcanzarla necesitamos un cambio de actitud, lo cual Acebal,2010, cita a Morales, 1990 que la define como "una predisposición aprendida, no innata, y estable aunque puede cambiar, a reaccionar de una manera valorativa, favorable o desfavorable, ante un objeto". (p.66) Sin embargo, para poder cambiar esa actitud se necesita primeramente tener conciencia ambiental por lo que existen diferentes enfoques de investigación como enfoque de conducta, enfoque mentalista o teorías cognitivas, enfoque funcionalista, enfoque genético o interaccionista unidos a los modelos de aprendizaje que se están dando actualmente. 
A su vez, la ONU declara el período del 2005-2014 como la Década de la Educación para el Desarrollo Sostenible, por lo que los gobiernos han estado llevando a cabo diversas campañas y acciones para ir mejorando la educación ambiental en la población tales como: la protección de los ecosistemas naturales, retribución por la conservación de los servicios ambientales, el hoy no circula, paseos ciclistas, entrega de focos ahorradores, entre muchos otros (Blanco, s.f.), por consiguiente el objetivo de esta investigación es evaluar la Conciencia Ambiental de los habitantes de la colonia Emilio Portes Gil en la $\mathrm{H}$. Matamoros (Tamaulipas) con cinco categorías, entendiéndose según la Real Academia Española como muy consciente: persona que sabe realmente que siente, piensa, quiere y obra con conocimiento de lo que hace; consciente: persona que siente, quiere y obra con conocimiento de lo que hace; medio consciente: es una persona que piensa, que siente y obra pero no con conocimiento pleno del tema; poco consciente: es una persona que piensa, que siente y obra pero no tiene muy definido su conocimiento hacia al tema a tratar; nada consciente: es una persona que piensa, que siente y que obra pero sin conocimiento del tema.

\section{METODOLOGÍA}

La muestra estuvo constituida por 50 habitantes de la colonia Emilio Portes Gil, de ambos sexos con un rango de edad de 8 a 68 años y estado civil de: soltero(a), casado(a), viudo(a), divorciado(a), madre soltera y unión libre.

El estudio que se realizó, fue tipo exploratorio-descriptivo, transversal con un enfoque cuantitativo, donde se recopiló información por medio de un instrumento llamado "Cuestionario de Actitudes Ambientales (CAAM) de Nuévalos", anteriormente usado en la Universidad de Valencia, el cual evalúo las conductas ambientales, donde se tuvo como variable independiente el conocimiento ambiental y como variable dependiente la conducta hacia el medio ambiente. Este instrumento consta de 17 ítems con respuesta tipo Likert y con cinco opciones de respuesta que van desde muy de acuerdo, de acuerdo, semi-acuerdo, en desacuerdo y muy en desacuerdo, la cual contiene cinco dimensiones que se observan en la Tabla 1 y se presenta el rango que le corresponde a cada una.

Tabla 1. Categorías de conciencia ambiental con los rangos correspondientes

\begin{tabular}{|l|c|}
\hline Categorias & Rango \\
\hline Muy consciente & $85-69$ \\
\hline Consciente & $68-52$ \\
\hline Medio consciente & $51-35$ \\
\hline Poco consciente & $34-18$ \\
\hline Nada consciente & $17-0$ \\
\hline
\end{tabular}

El cuestionario se aplicó en forma colectiva, en donde se acudió a la casa de los habitantes de la colonia Emilio Portes Gil para la recolección de datos, donde se leyó las instrucciones en cada casa. El tiempo estimado para su aplicación fue en un rango de 10-15 minutos. El análisis de los datos se realizó mediante el paquete estadístico SPSS versión 17.0. 


\section{RESULTADOS}

En los resultados encontramos que la población está conformada por un $52 \%$ de mujeres y un $48 \%$ de hombres, teniendo en la población de mujeres el rango mayor de $31 \%$ en las edades de 20-31 años y en los hombres un mayor rango entre los 8-19 años con un 46\% (ver Tabla 2).

Tabla 2. Rango de edades con respecto al sexo

\begin{tabular}{|l|c|c|c|c|c|c|}
\cline { 1 - 5 } Edad & \multirow{2}{*}{$8-19$} & $20-31$ & $32-43$ & $44-55$ & $56-58$ & Total \\
\cline { 1 - 6 } Sexo & & & & 5 & 3 & 26 \\
\hline Femenino & 4 & 8 & 6 & 5 & 2 & 24 \\
\hline Masculino & 11 & 4 & 2 & 10 & 5 & 50 \\
\hline Total & 15 & 12 & 8 & &
\end{tabular}

Se observó que existe muy poca diferencia de conciencia entre los hombres y las mujeres, ya que las mujeres reportan un $32 \%$ de conciencia ambiental y los hombres un $30 \%$. Otra de las categorías que se observa es que existe un $20 \%$ de medio consciente en las mujeres y en los hombres un $18 \%$, esto lo podemos observar en el Gráfico 1.

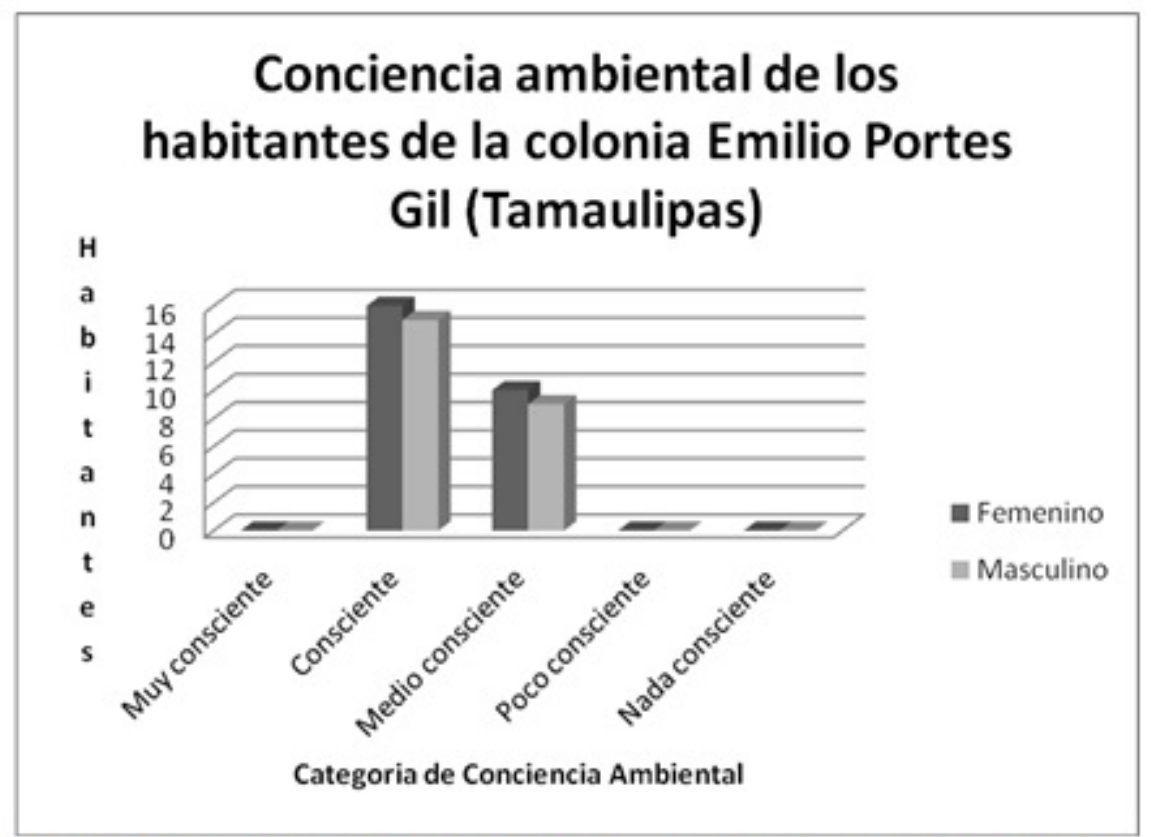

Gráfico 1. Conciencia ambiental de los habitantes de la colonia Emilio Portes Gil. (Tamaulipas)

Respecto al estado civil de la población, observamos en el Gráfico 2 un mayor porcentaje de habitantes casados con $44 \%$ de la población, y de estos detectamos que solo el $64 \%$ son de sexo femenino (ver Gráfico 2). 


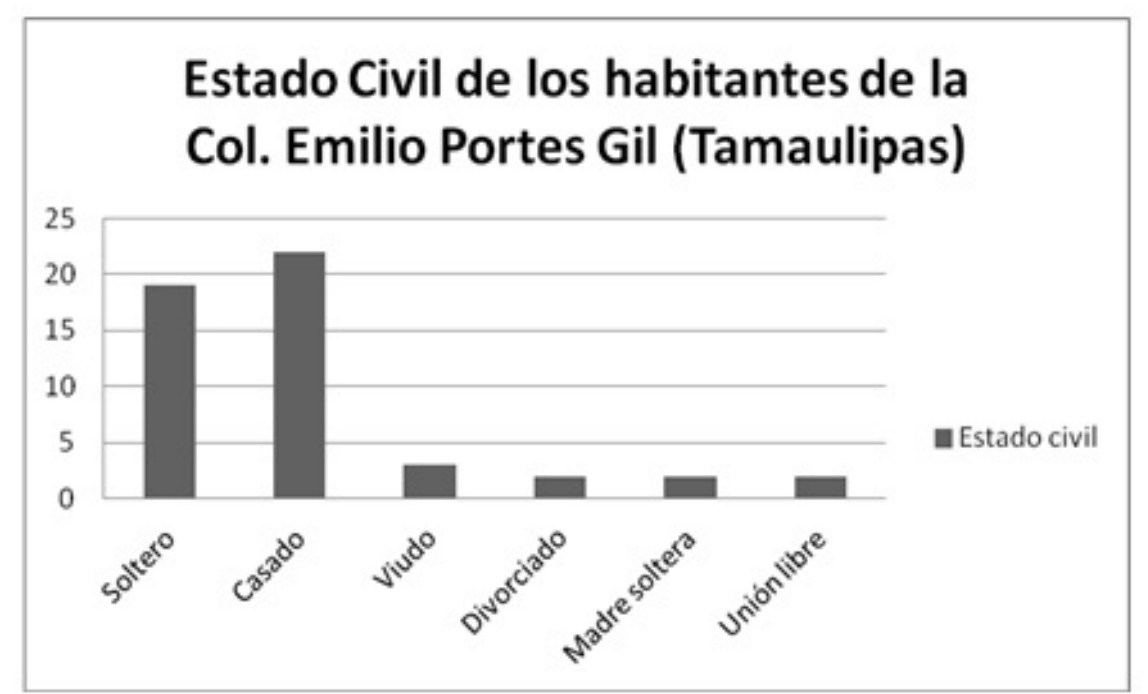

Gráfico 2. Estado civil de los habitantes de la colonia Emilio Portes Gil (Tamaulipas)

Relacionando el Gráfico 1 y el Gráfico 2, nos dice que existe mayor conciencia ambiental en el sexo femenino, pero tenemos que existe mayor población de casados sin saber aún a qué sexo se refiere, por lo que con el Gráfico 3 observamos que tenemos un $64 \%$ de sexo femenino. Además, se obtuvo un cálculo del coeficiente de Pearson con un valor de $P=0,347$, el cual indica que no existe diferencia entre el sexo de los habitantes respecto a la conciencia ambiental.

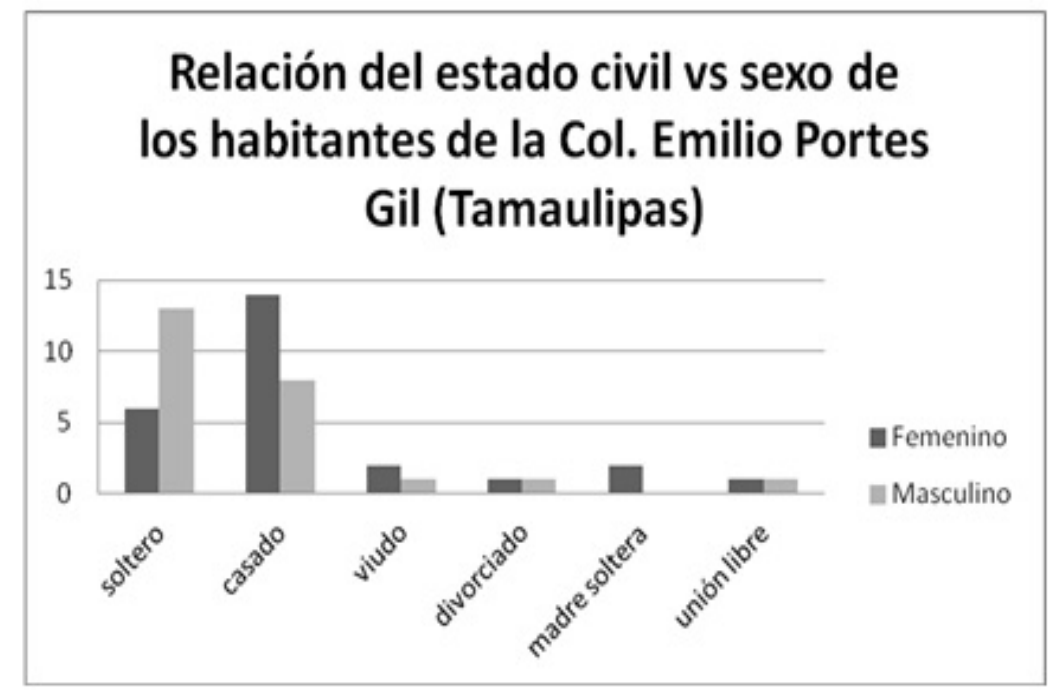

Gráfico 3. Relación del estado civil contra el sexo de los habitantes de la Col. Emilio Portes gil (Tamaulipas) 


\section{CONCLUSIONES}

El objetivo principal de esta investigación fue el de evaluar la conciencia ambiental de los habitantes de la colonia Emilio Portes Gil, observando que ningún sexo llegó alcanzar la categoría de "muy consciente".

Obteniendo el sexo femenino un $32 \%$ en la categoría denominada "consciente", y por el lado masculino un $30 \%$ en la misma categoría; lo cual nos indica que la población cuenta con conocimiento del impacto que se está generando en su entorno debido a sus actividades diarias, sin embargo no lo usa para obrar bien ante la Tierra.

A través de los resultados de este estudio se sugiere implementar programas ambientales en esa colonia como lo mencionan Vargas et al. (2011), para crear conciencia ambiental sobre la importancia del equilibrio naturaleza-hombre y la adquisición de actitudes y valores ambientales para poder mitigar los problemas existentes y obrar bien hacia el medio ambiente.

\section{AGRADECIMIENTOS}

A Luis Ángel Rosales Buenrostro, por su gran ayuda en la búsqueda de bibliografía, aplicación del instrumento y captura de datos.

\section{REFERENCIAS}

- Acebal, María del Carmen. (2010). Conciencia Ambiental y Formación de Maestras y Maestros (Tesis Doctoral). Universidad de Málaga, Facultad de Ciencias de la Educación. Disponible en: http://riuma.uma.es/xmlui/bitstream/handle/10630/4579/ TDR_ACEBAL_EXPOSITO.pdf?sequence $=6$

- Ávila, Alfredo. (s.f.). La educación ambiental a nivel superior. Universidad Autónoma de San Luis Potosí. Disponible en: http://www.bvsde.paho.org/bvsaidis/mexico13/052.pdf

- Blanco, Rafael. (s.f.). La conciencia ambiental. Revista Ambiente Plástico. Disponible en: http://www.sma.df.gob.mx/sma/links/download/biblioteca/ laconcienciaambiental.pdf

- Espejel, Adelina y Castillo, María. (2008). Educación Ambiental para el nivel medio superior: propuesta y evaluación. Revista Iberoamericana de Educación, 46(2). Disponible en: http://www.rieoei.org/2299.htm

- Molano, Carlos Alberto. (2003). Desarrollo de la conciencia Ambiental por medio de la lúdica. "Una propuesta pedagógica desde la educación ambiental para el desarrollo rural". Luna Azul. Disponible en: http://lunazul.ucaldas.edu.col index.php?option=com_content\&task=view\&id=65\&ltemid=65

- Moreno, Francisco Manuel. (2008). Origen, concepto y evolución de la educación ambiental. Revista Digital Innovación y Experiencias Educativas, 13. Disponible en: http://www.csi- 
csif.es/andalucia/modules/mod_ense/revista/pdf/ Numero_13/FRANCISCO_MORENO_1.pdf

- Real Academia Española. Disponible en: http://www.rae.es/rae.html

- Vargas, Catalina; Medellín, Juana; Vázquez, Laura y Gutiérrez, Gustavo. (2011). Actitudes Ambientales en los estudiantes de Nivel Superior en México. Luna Azul. Disponible en: http://lunazul.ucaldas.edu.co/index.php?option=content\&task= view\&id=648

- Vargas, Catalina; Vázquez, Laura; Gutiérrez, Gustavo; Vargas, María y Fernández, María. (2010). Conciencia ambiental en estudiantes de Enfermería Básica. Revista Universitaria de Investigación y Diálogo Académico, CONHISREMI, 6(3). Disponible en: http://conhisremi.iuttol.edu.ve/articles. php?code=PUBL000013

1. M.C. Directora, Instituto Tecnológico de Matamoros. Catedrática de Matemáticas y Desarrollo Sustentable. cathy_vargas@yahoo.com

Carr. Lauro Villar km 6,5 H. Matamoros, Tamps. Teléfonos 01(868) 81409 52 (868) 81409 53. Enviar correspondencia a: cathy_vargas@yahoo.com

2. Ing. Colaboradora, Instituto Tecnológico de Matamoros. Catedrática de Matemáticas. cynthiadinorah2006@hotmail.com

3. M.C. Colaboradora, Instituto Tecnológico de Matamoros. Catedrática de Química y Desarrollo Sustentable. socomancha@yahoo.com.mx

4. Q.F.B. Colaboradora, Instituto Tecnológico de Matamoros. Jefa del Departamento de Ciencias Básicas y Catedrática de Química y Desarrollo Sustentable.patricia_muzquiz@hotmail.com

5. Ing. Colaborador, Instituto Tecnológico de Matamoros. Jefe del Departamento de Ing. Civil y Catedrático de Hidráulica. avargas_gaxiola@hotmail.com

Para citar este artículo: Vargas, C., Briones, C. D., Mancha, M. del P. S., Músquiz, P. G. \& Vargas, A. (2013). Conciencia ambiental de los habitantes de la colonia Emilio Portes Gil en la H. Matamoros, Tamaulipas. Revista Luna Azul, 37, 155-161. Recuperado de http://lunazul.ucaldas.edu.co/index.php?option=content\&task=view\&id=850 